\title{
A New Approach to the Analysis of Short-range Order in Alloys using Total Scattering
}

\author{
L.R. Owen ${ }^{\mathrm{a}, \mathrm{b}}$, H.Y. Playford ${ }^{\mathrm{b},{ }^{*}}$, H.J. Stone ${ }^{\mathrm{a},}$ and M.G. Tucker ${ }^{\mathrm{b}, \mathrm{c}, 1}$ \\ ${ }^{\text {a }}$ Department of Materials science and Metallurgy, University of Cambridge, CB3 OFS, UK \\ ${ }^{\mathrm{b}}$ ISIS Facility, STFC Rutherford Appleton Laboratory, Didcot, Oxfordshire, OX11 0QX, UK \\ ${ }^{\mathrm{c}}$ Diamond Light Source Ltd, Harwell Science \& Innovation Campus, Didcot, Oxfordshire, OX11 0DE, UK
}
* Corresponding author. ISIS Facility, STFC Rutherford Appleton Laboratory, Didcot, OX11 0QX, UK. E-mail address: helen.playford@stfc.ac.uk (H. Y. Playford)
${ }^{1}$ Now at Spallation Neutron Source, One Bethel Valley Road, Oak Ridge, Tennessee, USA

\begin{abstract}
In spite of its influence on a number of physical properties, short-range order in crystalline alloys has received little recent attention, largely due to the complexity of the experimental methods involved. In this work, a novel approach that could be used for the analysis of ordering transitions and short-range order in crystalline alloys using total scattering and reverse Monte Carlo (RMC) refinements is presented. Calculated pair distribution functions representative of different types of short-range order are used to illustrate the level of information contained within these experimentally accessible functions and the insight into ordering which may be obtained using this new method. Key considerations in the acquisition of data of sufficient quality for successful analysis are also discussed. It is shown that the atomistic models obtained from RMC refinements may be analysed to identify directly the Clapp configurations that are present. It is further shown how these configurations can be enhanced compared with a random structure, and how their degradation pathways and the
\end{abstract}


distribution of Warren-Cowley parameters can then be used to obtain a detailed, quantitative description of the short-range order occurring in crystalline alloys.

Keywords: Atomic ordering; Diffraction; Pair correlation function; Short-range order; Short-range ordering 


\section{Introduction}

\subsection{Background and context}

The effects of short-range order on physical properties have long been noted in a variety of alloys systems. Thermodynamic discontinuities [1] in nickel chromium alloys, along with observed anomalies in electrical resistivity [2,3] have led to the description of a Komplex (K) state containing a different order to that expected for a random solid solution. Similarly, changes in magnetic properties, such as an increase in the observed spin wave stiffness of $\mathrm{Ni}_{3} \mathrm{Mn}$ [4], provide evidence for short-range ordering. In addition, short-range order has been shown to influence dislocation motion $[5,6]$ and has been observed in a number of systems used for structural applications, including nickel superalloys [7]. In spite of this influence on numerous materials properties, the study of short-range ordering in alloy systems has been largely abandoned over the last 20 years or so. This is due mainly to the lack of experimental evidence available from conventional diffraction experiments and the difficulties associated with the calculation of the ordering parameters from the diffuse scattering observed in single crystal experiments.

In this paper, a methodology is presented by which total scattering techniques may be used for the direct observation of short-range order in crystalline alloy systems. Simulated supercells based on the face-centered cubic $(f c c)$ structure, containing various types and degrees of short-range order, are used to explore the information content of pair distribution functions, which are experimentally accessible through Fourier transformation of total scattering data. The atomistic models that may be obtained from the analysis of such experimental data can used to provide quantitative information about the types of short-range order present, and approaches by which this can be achieved are discussed. 


\subsection{Total scattering}

Standard diffraction experiments, particularly of powder samples, derive structural information from the characteristic Bragg pattern [8]. Inherently, this analysis is based on the long-range average structure of the material and information on the short-range ordering that may be present in the system is lost in the averaging. The study of amorphous materials, which by definition lack the long-range order that leads to Bragg peaks, motivated the development of the total scattering technique, in which the Bragg and diffuse scattering from a sample are measured and analysed simultaneously. The technique has, more recently, been applied with great success to study many crystalline and disordered-crystalline systems [9], providing insight into the local information through analysis of deviations from the average structure. The basic scattering function for neutrons [10] is given as:

$$
S(Q)=\frac{1}{N} \sum_{i, j}\left\langle b_{i} b_{j} \exp \left(i Q \cdot\left[r_{i}-r_{j}\right]\right)\right\rangle
$$

where $i$ and $j$ are atomic labels, $r$ the instantaneous position of an atom, $b$ the atomic scattering length, $N$ the number of atoms and $Q$ the magnitude of the scattering vector $\boldsymbol{Q}$ i.e. the momentum transfer of the incident radiation. An equivalent expression for X-rays can be derived, replacing the neutron scattering length $b$ with the $Q$-dependent atomic form factor. For a periodic crystal this equation simplifies to the Bragg condition. In the case of an amorphous glass this may be written as:

$$
S(Q)=\frac{1}{N} \sum_{i, j} \bar{b}_{i} \bar{b}_{j} \frac{\sin \left(Q\left|r_{i}-r_{j}\right|\right)}{Q\left|r_{i}-r_{j}\right|}
$$

Separating the self-scattering components:

$$
S(Q)=\frac{1}{N} \sum_{i} \bar{b}_{i}^{2}+\frac{1}{N} \sum_{i \neq j} \bar{b}_{i} \bar{b}_{j} \frac{\sin \left(Q\left|r_{i}-r_{j}\right|\right)}{Q\left|r_{i}-r_{j}\right|}
$$

which can be recast in the form: 


$$
S(Q)=F(Q)+\sum_{i} c_{i} \bar{b}_{i}^{2}
$$

where $c_{i}$ is the concentration of species $i$ and the total scattering function, $F(Q)$, is given by:

$$
F(Q)=\rho_{0} \int_{0}^{\infty} 4 \pi r^{2} G(r) \frac{\sin Q r}{Q r} \mathrm{~d} r
$$

in which $\rho_{0}$ is the average density of the structure and:

$$
G(r)=\sum_{i, j} c_{i} c_{j} \bar{b}_{i} \bar{b}_{j}\left(g_{i j}(r)-1\right)
$$

where the $g_{i j}(r)$ terms are the partial pair distribution functions. The function $G(r)$ is often known as the pair distribution function (PDF). The inverse Fourier transform of Eq. 5, used for the calculation of a PDF, is given by:

$$
G(r)=\frac{1}{(2 \pi)^{3} \rho_{0}} \int_{0}^{\infty} 4 \pi Q^{2} F(Q) \frac{\sin Q r}{Q r} \mathrm{~d} Q
$$

Alternative normalisations of the PDF are sometimes used for convenience, to emphasise specific features of the function related to the properties and length-scales of interest. A full description of the other formulations of the PDF has been provided by Keen [11]. Unless otherwise stated, the terms $G(r)$ and PDF are used interchangeably here.

The partial PDFs are described mathematically as:

$$
g_{i j}(r)=\frac{n_{i j}(r)}{4 \pi r^{2} \rho_{i} \mathrm{~d} r}
$$

where $n_{i j}(r)$ is the number of atoms lying within $r$ and $r+\mathrm{d} r$, and $\rho_{i}=c_{i} \rho_{0}$, where $\rho_{0}$ is the density of the substance and $c_{i}$ the concentration of species $i$. Critically, this description is as applicable to periodic crystals as to amorphous materials.

The PDF, by definition, is a weighted histogram of the distribution of distances between atoms in the structure. A peak in the PDF will indicate the average distance of one atom relative to another; its width being dependent on the distribution of the inter-atomic distances 
(thermal vibration or static displacements) and its area being governed by the number and scattering length of the correlating atoms.

As is apparent from Eq. 5 and 7, the PDF and $F(Q)$ are effectively different representations of the same information (both contain the local structural information lacking in the Bragg peaks alone). Here the PDF is considered, as it is intuitively understandable as a visual representation of the local structure, but an equivalent analysis could be achieved through consideration of the $F(Q)$.

Whilst other local structural probes exist, such as EXAFS and NMR spectroscopy, they are of limited use for the analysis of short-range order in alloy systems, owing to the fact that ordering information for a large number of coordination shells can be extracted from a PDF, whilst EXAFS and NMR provide information for only the first couple of shells.

It is straightforward to achieve a qualitative understanding of the information provided by a PDF, however producing structural models is more involved. There are two popular approaches. The first, often known as 'small-box' modelling, utilises tools such as PDFgui [12], and is analogous to Rietveld refinement though the models are constrained by the PDF instead of the Bragg diffraction pattern. This technique is not well suited to the characterisation of short-range order in alloys since it produces a crystallographic description of a structure (i.e. one that comprises cell parameters, thermal parameters, fractional site occupancies), albeit one that is biased towards the local, rather than the average, structure. The second, a 'large-box' modelling technique using the reverse Monte Carlo [13] algorithm, is arguably better suited for the analysis of ordering in alloy systems as it is unconstrained by symmetry and produces large ( $>10,000$ atom) models that provide the ability to probe the ordering across appropriate length scales. 


\subsection{The reverse Monte Carlo technique}

Traditionally, analysis of total scattering data involves visual inspection of the scattering functions and the use of peak fitting to obtain information about the arrangement of the first one or two coordination shells. It is also common to use molecular dynamics or hard-sphere Monte-Carlo to calculate structural models, from which theoretical total scattering functions can be calculated and compared with the observed data. Analysis of the system is then possible only if the two are found to be in good agreement. The tuning of initial potentials to create a structural model that more accurately reflects the data is theoretically possible, but the direct effect of the potentials on the diffuse scattering is often difficult to quantify and the process of fine-tuning is laborious. To this end, the empirical potential structure refinement (EPSR) technique [14] combines direct Monte-Carlo simulations with the refinement of an empirical potential in order to obtain calculated scattering functions that are in good agreement with the observed data. In contrast, the reverse Monte Carlo (RMC) technique [13] is an iterative process in which goodness-of-fit parameters, which describe the statistical quality of the agreement between the model and the experiment, are minimised.

In the RMC method, an initial arrangement of atoms is created that reflects the average structure of the system, as determined by analysis of the Bragg diffraction data. The PDF calculated from this will consist of a series of delta functions, as every atom will be on its ideal position, with no allowance made for positional variations associated with thermal vibrations. However, a more realistic initial model can be obtained by applying small off-site displacements to each atom according to a pseudo-random Gaussian distribution generated using the Box-Muller method [15]. From this, a number of scattering functions may then be calculated and compared with the experimental data.

In general, the quality of the fit is defined by an agreement factor, $\chi^{2}$, which is calculated as follows: 


$$
\chi^{2}=\sum_{j}\left(y_{j}{ }^{\exp }-y_{j}{ }^{\text {calc }}\right) / \sigma_{j}^{2}
$$

where $j$ indicates a data point, $y_{j}$ exp the experimental value at that point, $y_{j}^{\text {calc }}$ the value calculated from the refined box and $\sigma_{j}$ the weighting factor for an individual data set. The $\chi^{2}$ parameters for different data sets are then summed as follows:

$$
\chi_{\mathrm{RMC}}^{2}=\chi_{F(Q)}^{2}+\chi_{G(r)}^{2}+\chi_{\text {Bragg }}^{2}+\chi_{\text {potentials }}^{2}+\cdots
$$

Any number of data sets can be summed, allowing the independent fitting of multiple different data sets simultaneously. The implementation of physical constraints (e.g. $\chi_{\text {potentials }}^{2}$ ) helps to ensure physically realistic results are obtained from the refinement.

The refinement makes random atomic translations and swaps and calculates the change in the scattering functions that result. Changes that lower $\chi_{\text {RMC }}^{2}$ will be automatically accepted, whilst those that increase $\chi^{2}{ }_{\text {RMC }}$ by $\Delta \chi^{2}{ }_{\text {RMC }}$ will be accepted with a probability of:

$$
P=\exp \left(-\frac{\Delta \chi^{2}{ }_{\mathrm{RMC}}}{2}\right)
$$

By accepting a certain number of 'bad' moves, the system may be prevented from becoming stuck in false minima. The result of the RMC refinement is an atomistic model that adequately fits all datasets and obeys all applied constraints.

The RMC algorithm has been implemented in a number of software packages (for example $[16,14,17,18])$ for the analysis of local structure and disorder. However, for accurate analysis of the structure of crystalline alloy systems, it is necessary to explicitly fit the Bragg pattern as well as the total scattering data. The RMCProfile [16] code, developed and maintained by some of the authors of this work, which incorporates this functionality, is therefore optimised for studies of this nature. 


\section{Background metallurgical theory}

\subsection{Super-lattice structures in binary alloys}

The phase diagrams of many alloy systems show regions where long-range ordered structures form, that differ from the disordered parent phases only in that the atoms are not randomly distributed but occupy specific lattice sites, either within a single cell or a supercell of the parent phase. This results in the breaking of the symmetry of the parent structure and the appearance of super-lattice peaks in the diffraction pattern. To demonstrate the efficacy of analysing short-range order though characterisation of pair distribution functions, the super-lattice structures of the face centred cubic $(f c c)$ lattice have been considered, but the analyses carried out could equally be extended to other structural families. Owing to the common occurrence of certain long-range ordered structures, Strukturbericht notation is normally adopted for ease of reference. For example the $\mathrm{L}_{2}$ structure $\left(\mathrm{A}_{3} \mathrm{~B}\right)$ is based on the face centred cubic structure, but with atoms of type A on the faces and atoms of type B on the corners. The case example for this type of ordering is $\mathrm{Cu}_{3} \mathrm{Au}$ [19], however it is common in other systems such as $\mathrm{Ni}_{3} \mathrm{Al}[20]$.

\subsection{Short-range order in binary alloys}

There are three main types of short-range order (shown in Fig. 1): statistical order, the disperse model and the micro-domain model. Statistical order is akin to the ordering observed in some glasses, where the proportion of atoms in a given coordination shell differs from that of the nominal stoichiometry. The statistical ordering model describes a homogenous order throughout the sample.

The alternative models describe heterogeneous short-range order, in which the order is not continuous, but rather nanoscale ordered regions exist within a disordered matrix. For systems such as $\mathrm{Cu}-\mathrm{Al}$ [21] the ordered regions are observed to be of a different stoichiometry to that of the bulk material, with the matrix compensating accordingly - an 


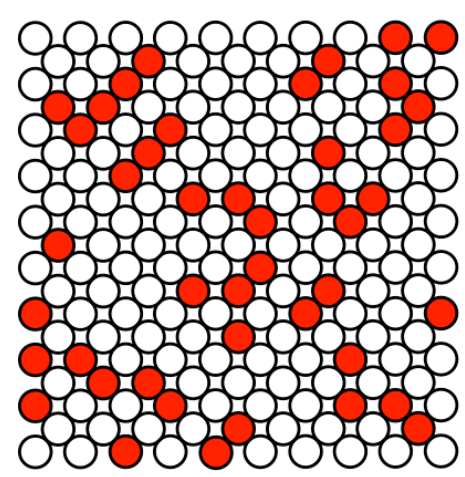

STATISTICAL

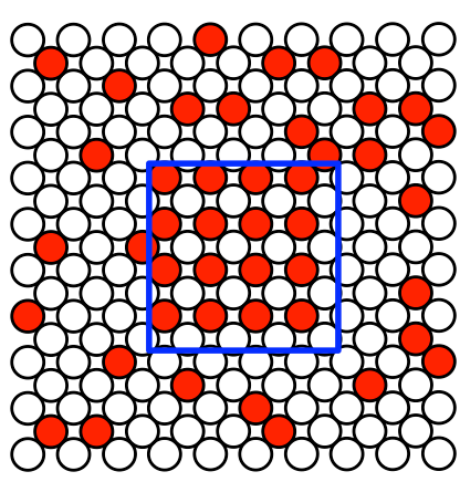

DISPERSE

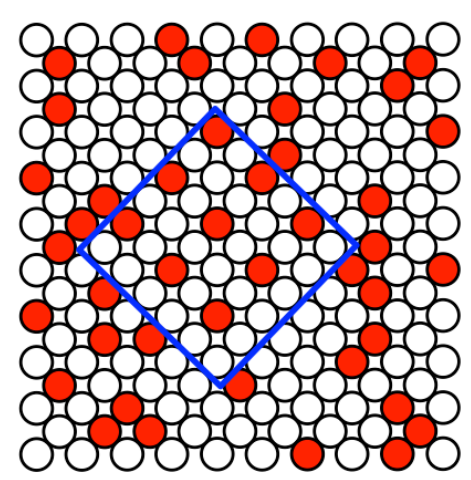

MICRO-DOMAIN

Fig. 1. Examples of the different types of short-range order that can be exhibited by a crystalline alloy system. In each case the average unit cell of each box is the same. In the disperse and micro-domain models, the ordered region is outlined by the solid line.

example of the disperse model. This model also describes clustering of atoms, such as that observed in the $\mathrm{Cu}-\mathrm{Ni}$ system [22]. Alternatively, the $\mathrm{Cu}_{3} \mathrm{Au}$ system exhibits short-range ordering consistent with the micro-domain model $[23,22]$, with the ordered regions and matrix being of the same stoichiometry and only distinguishable by the level of order present.

Whilst the formation of a random solid solution is driven by entropic effects, long-range ordered structures are driven by the favourable enthalpy of forming like or dislike bonds in a given coordination shell. Short-range order exists between these two extremes as a balance between increased enthalpy of forming certain favoured bonds and the loss in entropy caused by doing so. Therefore, it is necessary to consider both of these end member states in any future analyses in order to understand the intermediate short-range ordered systems.

\subsection{Quantitative descriptions of short-range order}

In order to link the short-range order of a system to its observed physical properties, quantitative descriptors of the ordering occurring in the system are required. This section 
outlines two of the main methods by which this is typically achieved. The way they can be related to large box models, is discussed in Section 3.3.

\subsubsection{Warren-Cowley parameters}

The Warren-Cowley parameters $[24,25]$ are one of the simplest and oldest methods of describing short-range order in a system and are most closely linked to the statistical model of short-range order. They describe how the observed occupation of a coordination shell differs from that of the nominal stoichiometry.

They are defined as:

$$
\alpha_{l m n}^{A B}=1-\frac{P_{l m n}^{A B}}{c_{B}}=1-\frac{P_{l m n}^{B A}}{c_{A}}
$$

where $P_{l m n}^{A B}$ is defined as the probability of finding a B atom at position $\vec{r}_{l m n}=l \vec{a}_{1}+m \vec{a}_{2}+$ $n \vec{a}_{3}$ (where $\vec{a}_{1}, \vec{a}_{2}$ and $\vec{a}_{3}$ are lattice vectors, and $l, m$ and $n$ are fractional coordinates) from an origin placed on an $\mathrm{A}$ atom, and $c_{A}$ and $c_{B}$ are defined as the atomic fractions of $\mathrm{A}$ and $\mathrm{B}$ respectively in the alloy. For a random distribution of atoms in an alloy, $\alpha_{\operatorname{lm} n}^{A B}=0$, with the exception that, by definition, for any system $\alpha_{000}^{A B}=1$. Positive values (to a maximum of 1 ) suggest a preference for like atom coordination in the system; conversely negative values (to

a minimum value of $\frac{c_{A}-1}{c_{A}}$ when measured around $\mathrm{B}$, and $\frac{c_{B}-1}{c_{B}}$ when measured around $\mathrm{A}$ ) indicate a preference for dislike atom coordination.

Whilst for a random structure all $\alpha^{A B}$ parameters will tend to zero, for long-range ordered structures, $\alpha^{A B}$ will oscillate in a predictable manner through the coordination shells. Historically, experimental $\alpha$-parameters were calculated using either the Borie-Sparks [26], Georgopolous-Cohen [27] or $3 \lambda$ methods [28], from single crystal diffuse scattering patterns. These reported values are the average $\alpha$-parameter of the sample.

Whilst the preceding description applies only to binary alloy systems, extensions have been made for ternary or higher systems. For higher-dimensional systems, ordering 
parameters analogous to the Warren-Cowley parameters are possible, such as the pairwise multicomponent short-range order (PM-SRO) parameters [29], which are defined as:

$$
\alpha_{m}^{A B}=\frac{p_{m}^{A B}-c_{B}}{\delta^{A B}-c_{B}}
$$

where $\delta^{A B}=1$ if $A=B$ and zero otherwise, $p_{m}^{A B}$ is the probability of finding a B-type atom around an A type atom, in shell $\mathrm{m}$. This parameter reduces to the Warren-Cowley parameter for a binary alloy. More complex formulations, such as the generalised multicomponent short-range order (GM-SRO) parameters [30], also exists that can describe interactions of multiple species.

\subsubsection{Clapp configurations}

Although they provide useful information about the hallmarks of short-range order in a system, the Warren-Cowley parameters do not provide an exact description of local atomic arrangements within an alloy. As an alternative description, Clapp [31] published a complete list of the 144 possible arrangements of the 12 nearest neighbour atoms in an $f c c$ lattice. The number of configurations is reduced from the theoretical maximum of 4096 by the removal of any orientation basis of the configuration.

An alloy with a random distribution of atoms will occupy many Clapp configurations, whereas a long-range ordered structure will occupy only a range of very specific configurations that are necessary structural motifs for the building of the structure. Fig. 2 shows three common long-range ordered structures and the associated Clapp configurations from which they are made. 


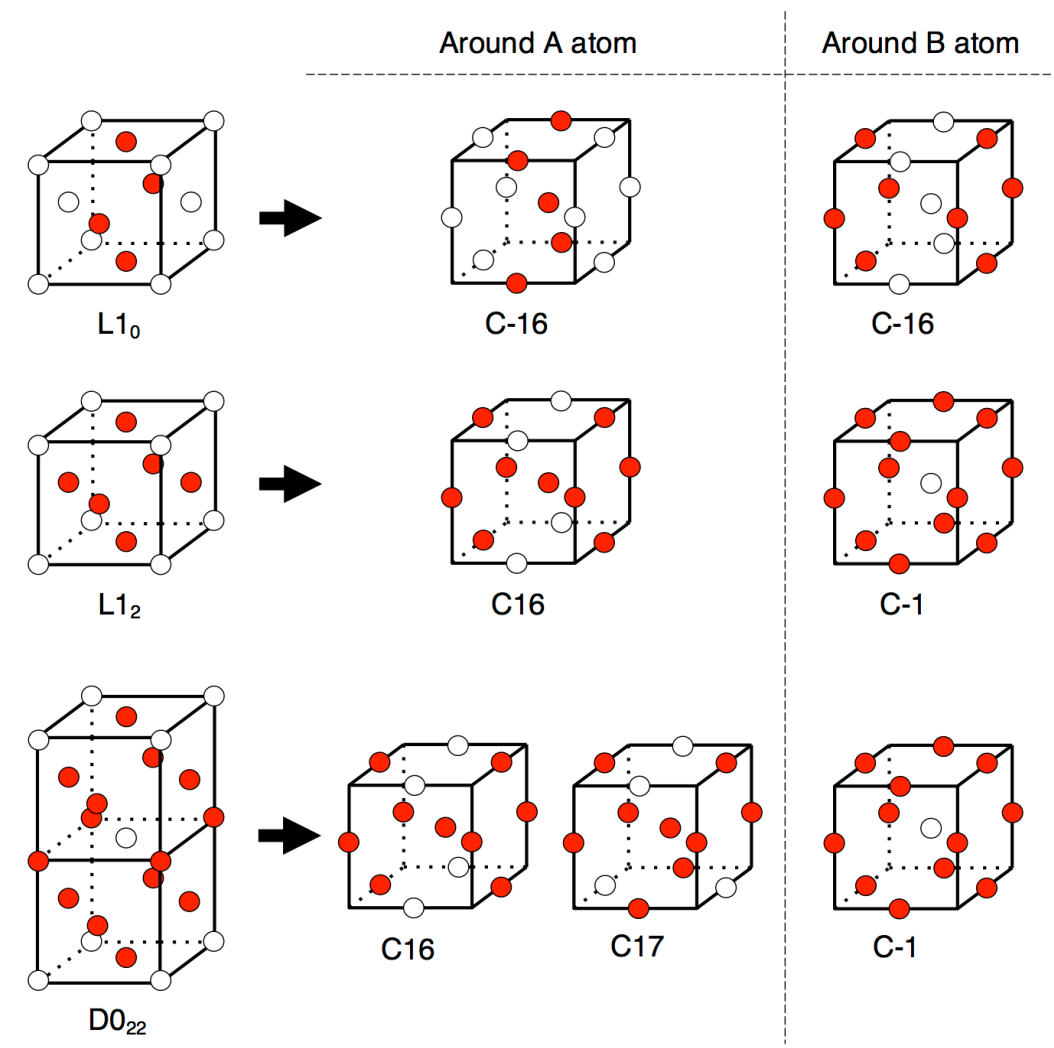

Fig. 2. Some typical long-range ordered structures and the Clapp configurations occupied by the atoms in the structure. Filled circles represent atoms are of type $\mathrm{A}$ and unfilled circles atoms of type $\mathrm{B}$. $\mathrm{L} 1_{0}$ has stoichiometry $\mathrm{AB}$, whilst $\mathrm{L}_{2}$ and $\mathrm{D} 0_{22}$ are both $\mathrm{A}_{3} \mathrm{~B}$.

3. Development of total scattering analysis for metallurgical systems

\subsection{Experimental considerations}

\subsubsection{The measurement of high-quality PDFs}

Owing to the low intensity of the diffuse scattering signal, compared with the much stronger Bragg peaks, high quality data are required in order to obtain a statistically significant measurement of the diffuse scattering in a total scattering experiment. Care must be taken in both the acquisition of the data and the subsequent correction, processing and analysis.

It can be seen from Eq. 7 above, that the integral should be calculated over an infinite $Q$ range, and while this is obviously not achievable experimentally, as wide a $Q$-range as 
possible is required for obtaining a high-quality PDF. Since the magnitude of $Q$ is given by $Q=\frac{4 \pi \sin \theta}{\lambda}$ (where $\theta$ is the diffracted angle and $\lambda$ the incident radiation wavelength) it can be seen that the use of short-wavelength incident radiation is desirable. A typical laboratory Xray diffractometer operating with $\mathrm{Cu} \mathrm{K}_{a}$ radiation can achieve a $Q_{\max } \sim 6 \AA^{-1}$, while a specialised instrument with an $\mathrm{Ag}$ source can achieve $Q_{\max } \sim 18-20 \AA^{-1}$. However, superior data may be obtained at high-energy synchrotron X-ray and spallation neutron sources, which can achieve a $Q_{\max }>30 \AA^{-1}$.

The choice between synchrotron X-ray and neutron radiation, should be made based on the specific system of interest. The radiation should be chosen so as to maximise the difference in the scattering between the random and ordered structures. This will be dependent on the relative concentrations of the atomic species and their relative scattering lengths and form factors. For example, the study of $\mathrm{Cu}-\mathrm{Au}$ alloys with neutrons is unfeasible due to their almost identical neutron scattering lengths; and conversely, the study of alloys with many components of similar atomic number would be impossible with X-rays.

\subsubsection{Sample preparation}

Total scattering is, by nature, a powder technique and so requires samples that are crystallographic powders. Therefore, when studying engineering materials and alloys the issue of crystallographic texture inherited from the prior processing must be accounted for. Whilst the effect of texture on Bragg data is well documented in the literature, the effect of texture on a PDF is not well understood. Hence, collected total scattering data must be as free from textural effects as possible, and the Bragg intensities should therefore be carefully assessed for discrepancies.

In addition to issues associated with the sample texture, the experimental set up should seek to maximise the number of crystallites illuminated by the incident radiation to ensure a powder average is achieved. For neutrons, a large sample volume makes this relatively 
straightforward; however, with X-rays the irradiated volume is much smaller, and care must be taken during sample preparation to minimise crystallite size. In alloy systems, thermomechanical processing is commonly used to reduce grain size but can result in strong crystallographic textures. Consequently, an atomisation technique (either water or gas) is required. In gas atomisation, the alloy is heated until molten and sprayed with argon to produce spherical droplets of powder. Water atomisation is similar but relies on a jet of water rather than argon, resulting in a greater variation in particle size and morphology. Gas atomisation is known to produce solidification induced micro-segregation textures in the gas atomised powder, so samples must be subsequently heat-treated in the single-phase field (solid solution) in order to remove multiple phases with different compositions and ensure a homogenous random sample is ultimately achieved. The powders made in this manner can subsequently be heat-treated at the temperature of interest to allow ordering to develop in the sample.

\subsection{Calculated PDFs}

For all of the proposed models of short-range order in alloy systems, the limiting cases for ordering in structures are the random distribution and long-range ordered structures. A comparison between the theoretical PDFs of these structures can be used to demonstrate the level of information available in a PDF, and what may be observed in short-range ordered systems.

To illustrate this, $20 \times 20 \times 20$ supercells of the face-centred cubic $(f c c)$ structure (with lattice parameter $a=3.55 \AA$ ) were created to reflect possible structures of the $\mathrm{Cu}_{3} \mathrm{Au}$ alloy at high temperature (atoms randomly distributed across all sites in the $f c c$ ) and low temperature (forming the $\mathrm{L}_{2}$ structure). A random displacement was applied to the atom positions to yield a Gaussian distribution that mimics the effect of thermal motion. The same Gaussian displacement was applied to both structures, the situation reflecting different degrees of order 
frozen into the structure at the same temperature by quenching. PDFs were calculated for these structures, as if the sample were an alloy of copper and gold scattering X-rays and are shown in Fig. 3.

It is important to note that, in contrast to systems more typically studied with total scattering, alloy ordering transitions are not necessarily associated with a shift in the peak positions or the appearance of additional features in the PDF. Instead, only the area of the peaks will change as they reflect alterations in the occupation of different coordination shells (see Eq. 8). Identifying which peaks change, and the extent of the change, can provide

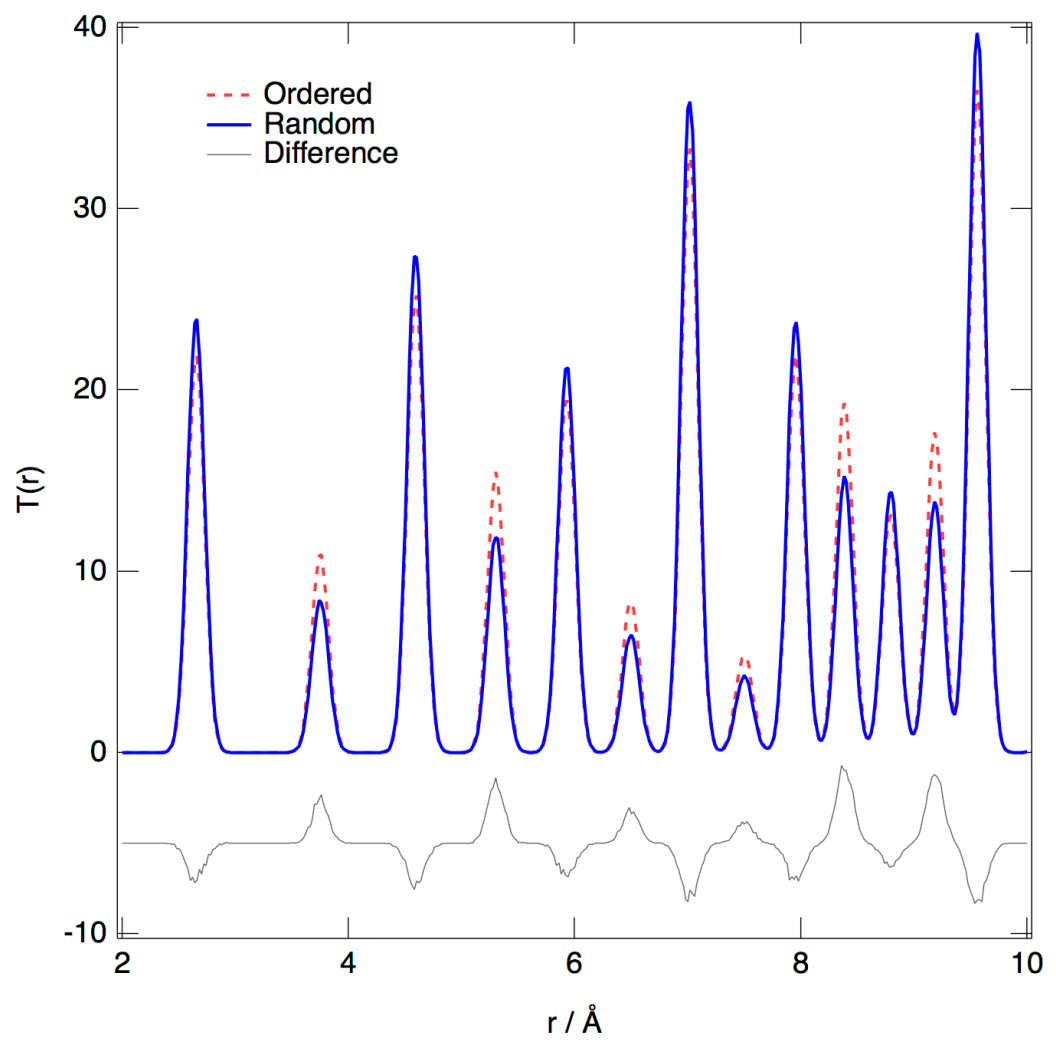

Fig. 3. Calculated PDFs for a random $\mathrm{Cu}_{3} \mathrm{Au}$ alloy (solid) and an ordered $\mathrm{L}_{2}$ (dotted) structure. The offset line indicates the calculated difference.

\footnotetext{
* It is common in the analysis of X-ray total scattering data to use an average atomic form factor as a simple method to account for the Q-dependency of X-ray scattering [33]. As such this effectively approximates the system as a series of "point-scatterers", equivalent to the scattering of neutrons. Therefore, in this study the PDFs have been calculated using a $\mathrm{Q}$ independent X-ray "scattering length" equivalent to the atomic number, Z, to weight the contributions of each pair of atoms.
} 
information about the type of ordering occurring in the system. This can be demonstrated by a consideration of a host of long-range ordered structures.

The number of long-range ordered structures is a countable infinity; however, here the consideration is restricted to a subset of structures, those with 4 atoms or fewer in the unit cell. Hart [32] suggested that there are only 17 binary supercells modelled on a $f c c$ structure that fulfil this criterion and listed those that have been observed and those that have yet to be observed in the literature. For reference, images of these structures can be found in Supplementary Fig. S1. The D1 $1_{\mathrm{a}}$ structure is also included for consideration, as it is part of a family of structures commonly observed in short-range ordered systems.

To examine the sensitivity of the PDF to different types of ordering, $24 \times 24 \times 24$ facecentred cubic $(f c c)$ supercells were created of the eighteen long-range ordered structures of interest, in a similar manner to that outlined previously (for the $\mathrm{D} 1_{\mathrm{a}}$ a $25 \times 25 \times 25$ box was used owing to the dimensions of the $\mathrm{D} 1_{\mathrm{a}}$ supercell). PDFs were calculated in the same manner as previously. The atomic arrangement was randomised using unconstrained MonteCarlo swapping and the normalised PDF calculated for the random structure. The difference between the ordered structure PDF and that of the randomised box was calculated, and these difference PDFs, which represent the maximum differences that can be expected to occur, are shown in Fig. 4.

It is important to note that the predicted difference PDFs shown in Fig. 4 represent the differences between long-range order and no order. To extend this analysis to the study of short-range order, it is necessary to consider how the PDF would vary depending on the different types and amounts of short-range order that could occur. To this end, a Monte-Carlo algorithm was written to generate statistically ordered boxes with specified Warren-Cowley parameters. Software was also written to generate supercells containing different size domains of long-range ordered structures, to create microdomain and disperse-model based 

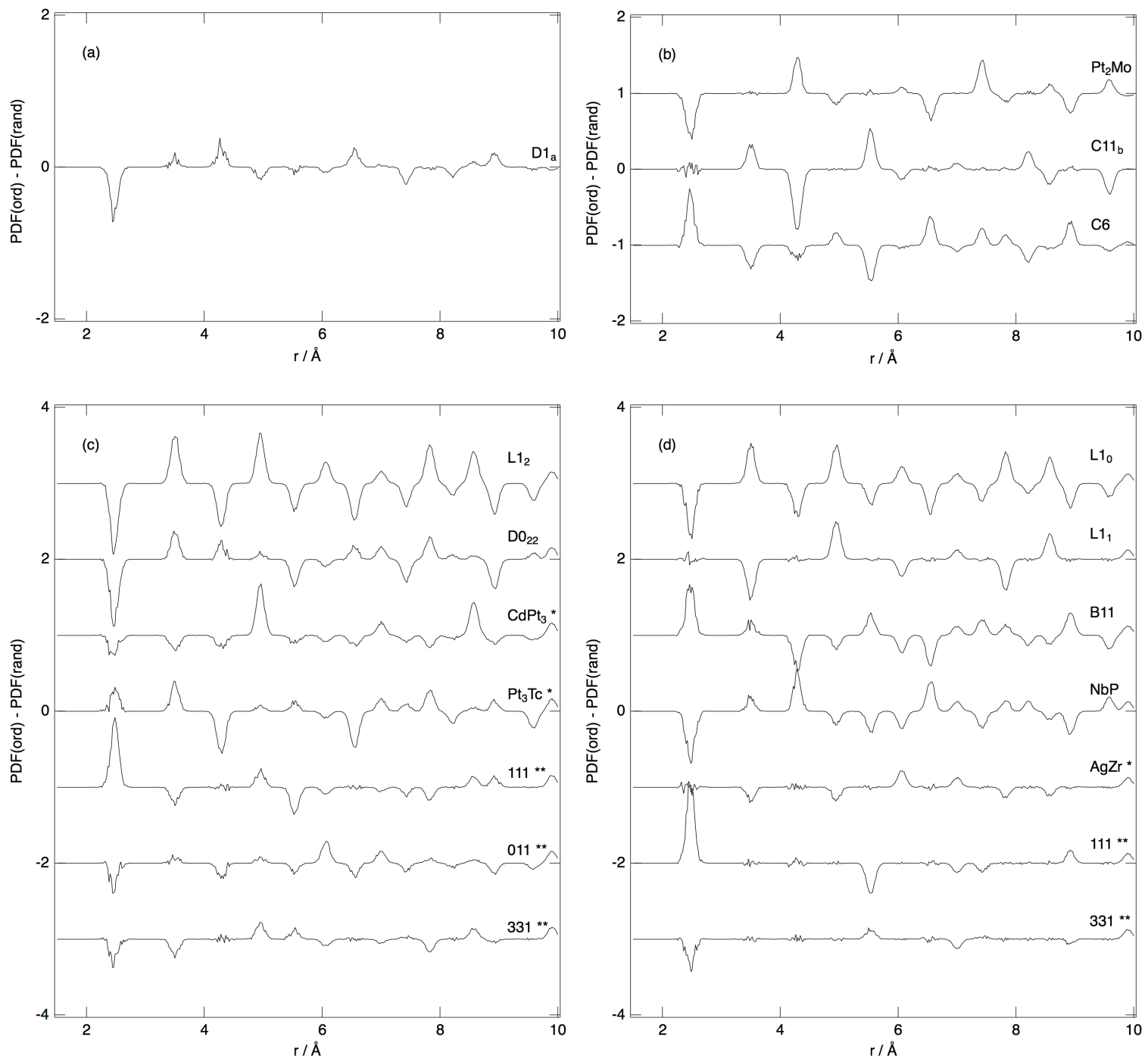

Fig. 4. Predicted difference PDFs for some long-range ordered binary alloy structures. The panels reflect long-range ordered structures of the same stoichiometry: (a) $A_{4} B$, (b) $A_{2} B$, (c) $\mathrm{A}_{3} \mathrm{~B}$ and $(\mathrm{d}) \mathrm{AB}$. The structures are those described by Hart [32] and the $\mathrm{D} 1_{\mathrm{a}}$ structure. Images of all the structures can be found in the figure S1 for reference. Structures indicated by * have been predicted to exist in the system they are named after, but as yet have not been physically observed. Structures indicated ** have been neither predicted nor observed. These are named after the family of planes in which there is an observed oscillation in the type of atom occupying the plane. Otherwise structures are named after with the standard Structurbericht notation, or the case example system in which they are observed to exist.

systems. A detailed explanation of the generated boxes can be found in the supplementary information. 
Given that the change in area of the peaks is of greatest interest, as previously mentioned, the integral of the PDFs is calculated and the difference from a random arrangement of the same nominal stoichiometry (i.e. the $n=0$ or $m=0$ case) plotted, Fig. 5.

Whilst for the statistical and microdomain models the difference integral PDF oscillates close to the base line, for the disperse model there is a notable increase in the base line with size of domain. This is due to differences between the local concentration and the nominal stoichiometry of the system. Oscillations on this curved background are also visible that have a similar dampening to that observed in the microdomain case. For the microdomain system, as the size of the domain increases, so does the magnitude of the oscillations, and the degree of dampening decreases (i.e. the number of shells in which the oscillation is observed increase) as the domain gradually grows to fill the box completely. A similar effect of
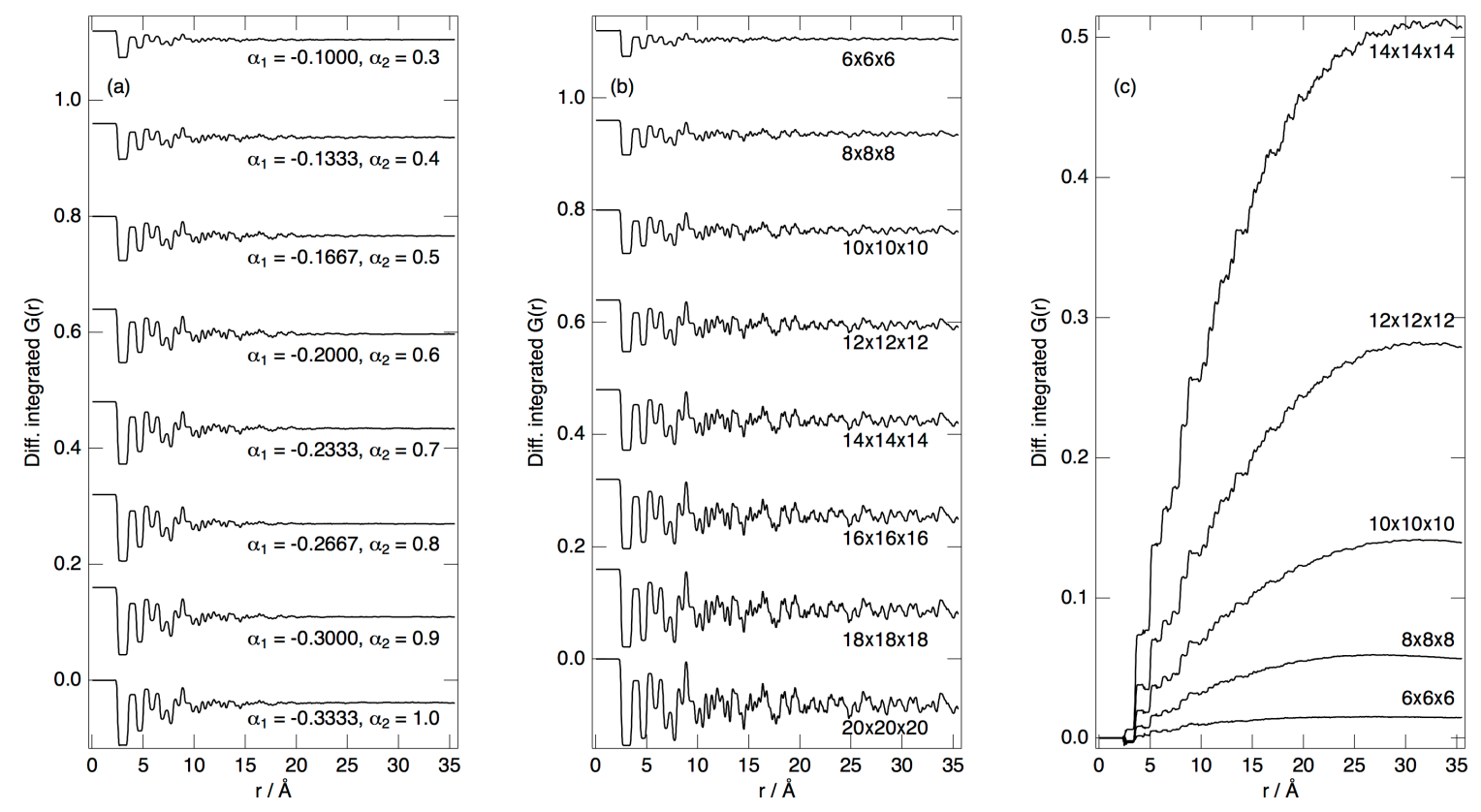

Fig. 5. Difference integrated PDFs for a series of boxes containing different types and amounts of short-range order. The panels are as follows: (a) Statistical order with different magnitudes of Warren-Cowley parameter for the first two shells (created by a Monte-Carlo simulation), (b) Microdomain order - containing cubic domains of the $\mathrm{L}_{2}$ structure of different sizes, (c) Disperse order - containing cubic domains of the $\mathrm{L} 1_{0}$ structure of different sizes. In panels (a) and (b) the difference integral PDFs are offset for ease of viewing. 
increased magnitude of oscillation is notable in the statistically ordered case. However, the change in the number of affected shells is relatively constant and does not change as much as the micro-domain case.

\subsection{Extracting short-range order parameters from structural models}

\subsubsection{Warren-Cowley extension to large box models}

Warren-Cowley parameters can be extracted directly from an atomistic model, by averaging the Warren-Cowley parameter calculated for every individual atom. From the set of long-range ordered structures used to simulate the PDFs in Fig. 4 it is possible to calculate how the Warren-Cowley parameters oscillate through the co-ordination shells. The calculated $\alpha^{A B}$ parameters for some standard structures are shown in Supplementary Fig. S2.

Similarly, the Warren-Cowley parameters for the short-range ordered boxes generated previously can be calculated. A selection of these, corresponding to both the disperse and microdomain cases, are shown in Supplementary Fig. S3. It can be seen that the magnitude of the oscillation changes as expected. However, it is difficult to assign any meaningful interpretation to the oscillations.

More usefully, by using a large box method it is possible to extract additional information about the system by a consideration of the distribution of the Warren-Cowley parameters within a given shell, and not simply the average value for each shell for the entire box. For a random box, the theoretical distribution of the Warren-Cowley parameters in a given shell may be calculated by a simple application of binomial theorem.

For a given atom the probability, $P\left(n_{A}\right)$, of it having $n_{A}$ neighbouring atoms of type $\mathrm{A}$, in a coordination shell containing $d$ atoms is given as:

$$
P\left(n_{A}\right)=\left(\frac{d !}{n_{A} !\left(d-n_{A}\right) !}\right) c_{A}^{n_{A}} c_{B}^{d-n_{A}}
$$

where $c_{A}$ and $c_{A}$ are the concentration of $A$ and $B$ respectively. For a given value $n_{A}$ the Warren-Cowley parameter can then be calculated as: 


$$
\alpha^{A B}=1-\frac{n_{A}}{d c_{B}}
$$

For every atom in the model, the Warren-Cowley parameter may be calculated, and so the distribution of the number of atoms with a given parameter plotted. By comparing these observed distributions, in a given shell, with those predicted by the binomial distribution above, information about the nature and magnitude of order in the box may be extracted.

The distributions of Warren-Cowley parameters from different models of short-range order can be calculated using the same simulated boxes as previous. Fig. 6 shows the first shell Warren-Cowley parameters for the random case and different simulated types of shortrange order.

The observed Warren-Cowley parameters in the random case (a) can, as expected, be seen to agree well with those predicted using the binomial theorem. In the statistical case (b) it can be seen that, while the general shape has been maintained, the maximum of the distribution has shifted. This reflects the fact that, on average across the box, A atoms tend to form more A-B bonds in their first coordination shell than would be expected based on the stoichiometry alone. In the microdomain box (c), the contribution of the random matrix (dotted line) has been scaled by a factor of 0.875 to reflect the proportion of the box it occupies, and this reveals a clear increase in the number of atoms (both A and B) with $\alpha_{1}=-$ 0.3333 , corresponding to the atoms in the $\mathrm{L}_{2}$ microdomain region. Finally, in the $\mathrm{L} 1_{0}$ disperse box (d), increases in different parameters for the two atom types $\left(\alpha^{\mathrm{AB}}=-1.6667\right.$ and $\left.\alpha^{\mathrm{BA}}=0.1111\right)$ are apparent, which is indicative of the stoichiometry of the domain being different to that of the box. In this case, the matrix contribution was scaled for both the proportion of the box it occupies and the difference in concentration caused by the disperse domain. 

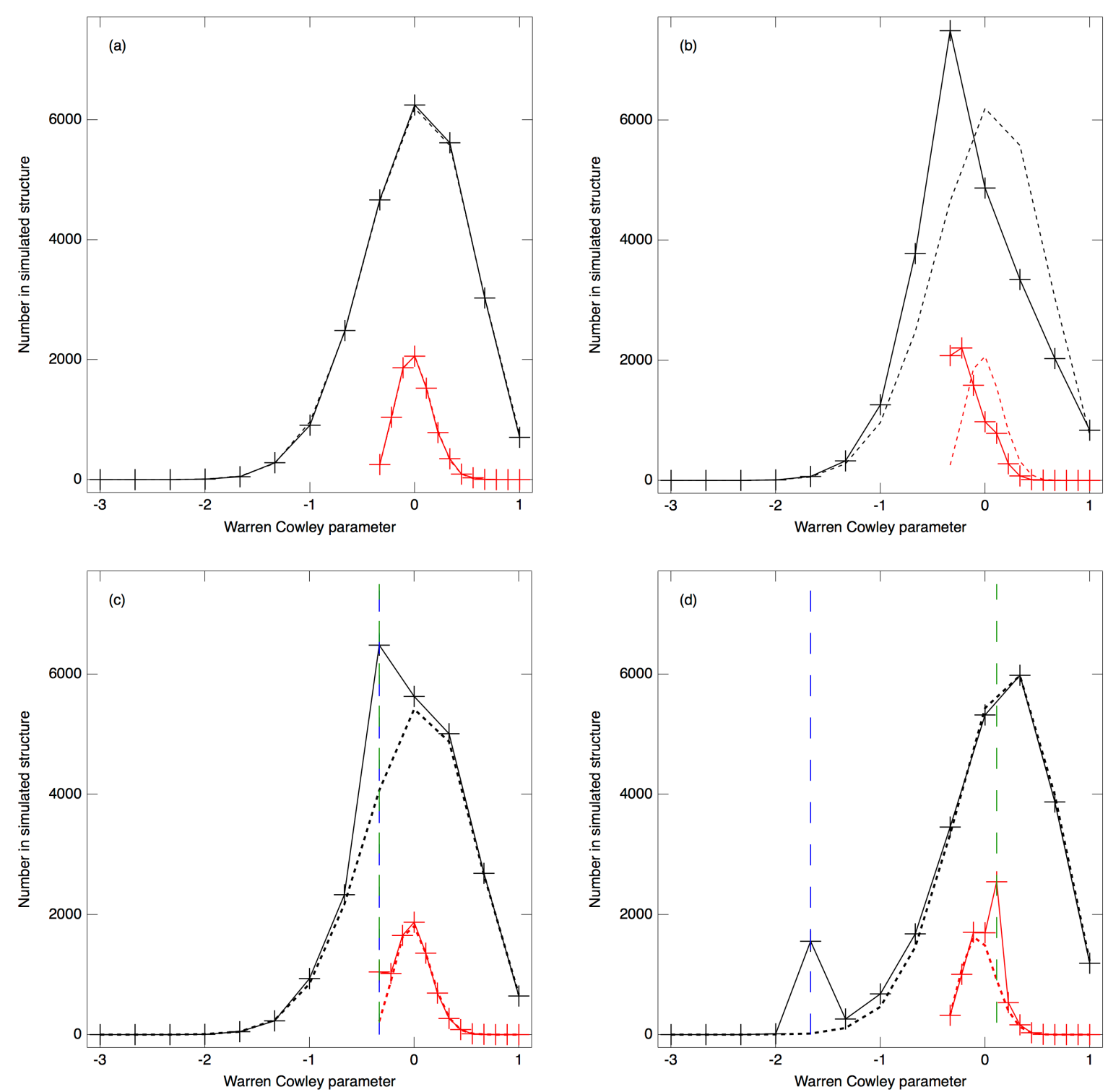

Fig. 6. The distribution of Warren-Cowley parameters for the first coordination shells $\left(\alpha_{1}\right)$ in generated supercells of size $20 \times 20 \times 20$ and stoichiometry $\mathrm{A}_{3} \mathrm{~B}$. The short range order present is as follows: (a) random, (b) statistical order created using a Monte Carlo simulation with a preference for dislike nearest-neighbour bonds (average $\alpha_{1}{ }^{\mathrm{AB}}=-0.0417$ and $\alpha_{2}{ }^{\mathrm{AB}}=0.1250$ ), (c) an $\mathrm{L}_{2}$ microdomain of size $10 \times 10 \times 10$ unit cells and (d) an $\mathrm{L} 1_{0}$ disperse domain of size 10x10x10 unit cells. In all cases, the solid black line with markers indicates the observed values around $\mathrm{A}$ atoms $\left(\alpha_{1}^{\mathrm{AB}}\right)$. The solid red line with markers indicates the observed values around $\mathrm{B}$ atoms $\left(\alpha_{1}{ }^{\mathrm{BA}}\right)$. For panels (a) and (b) the dotted lines indicate the contribution of the random matrix distribution predicted by Eq. 14 and 15. For panels (c) and (d) the dotted lines indicate the random distribution for the matrix contribution as predicted by Eq. 14 and 15 and accounting for the change in local concentration of the matrix and size of the matrix compared with the size of the domain. In panels (c) and (d) an increase in occupation of a specific $\alpha_{1}{ }^{\mathrm{AB}}$ and $\alpha_{1}{ }^{\mathrm{BA}}$ are indicated by vertical dashed lines. 
By working out the concentration at which $\alpha^{\mathrm{AB}}=\alpha^{\mathrm{BA}}$ for the parameters with increased occupation, the concentration of the ordered domain, $c_{A, \text { dom }}$, can be calculated. i.e.:

$$
c_{A, \mathrm{dom}}=\frac{\left(1-\alpha_{\mathrm{obs}}^{B A}\right) c_{A, \mathrm{obs}}}{\left(1-\alpha_{\mathrm{obs}}^{B A}\right) c_{A, \mathrm{obs}}+\left(1-\alpha_{\mathrm{obs}}^{A B}\right) c_{B, \mathrm{obs}}}
$$

where $\alpha_{\mathrm{obs}}^{A B}$ and $\alpha_{\mathrm{obs}}^{B A}$ are the increased Warren-Cowley parameters in the observed distribution, and $c_{A, \mathrm{obs}}$ and $c_{B, \mathrm{obs}}$ are the nominal stoichiometry of the box. This can then be used to fit the remainder of the pattern with a matrix of the correct size and concentration, and thus estimate the size of the domain. When performed on the $\mathrm{L} 1_{0}$ disperse box considered previously, this calculation yields a domain size of approximately $9 \times 9 \times 10$ unit cells, very close to the correct value of $10 \times 10 \times 10$.

Whilst in these cases it is possible to see the increase in the number of atoms with an $\alpha$ corresponding to that of the ordered domain, as the domain size reduces, it becomes almost impossible to see this increase. For a $5 \times 5 \times 5$ unit cell domain, i.e. $1.5625 \%$ of the volume of the sample, it is no longer possible to see this increase and extract information about the system. The exact percentage at which this increase would not be visible is dependent on the stoichiometry of both the overall system and the local concentration in the domain.

Although here only the first shell has been considered, a similar analysis could be performed in all shells and the nature of ordering occurring in the microdomain extracted.

\subsubsection{Clapp Configurations from large box models}

In a large box model that represents an alloy with no long- or short-range order, the occupation of a given Clapp configuration will depend only on the stoichiometry of the alloy, the number of like atoms in the box and the multiplicity of the configuration. The theoretical probability of a given Clapp configuration $a$ existing in a face centred cubic alloy $\mathrm{A}_{x} \mathrm{~B}_{(1-x)}$ (around either atom type A or B) can be calculated using the binomial theorem and is given by: 


$$
p_{a}=m_{a}\left[x^{n}(1-x)^{13-n}+x^{13-n}(1-x)^{n}\right]
$$

where $n$ is the number of like atoms amongst the 12 nearest neighbours, $m_{a}$ is the multiplicity of the configuration and $x$ is the concentration of species A.

Comparing this theoretical distribution with several random supercells, the model is found to be in good agreement. The variation in occupation of a given configuration between the simulated random box and the values calculated from Eq. 17 should be within the statistical variation of the distribution. As such, it is essential to obtain some measure of this statistical noise, in order to separate the real enhancements from the random fluctuations of the box. Considering only one Clapp configuration and arguing that either an atom exists in this configuration, or does not, a second application of the binomial theorem states that the mean $\left(\overline{n_{a}}\right)$ and standard deviation $\left(\sigma_{n_{a}}\right)$ in occupation of the configuration $\left(n_{a}\right)$ will be given by:

$$
\begin{gathered}
\overline{n_{a}}=p_{a} N \\
\sigma_{n_{a}}=\sqrt{p_{a}\left(1-p_{a}\right) N}
\end{gathered}
$$

where $p_{a}$ is as defined in Eq. 17 and $N$ the number of atoms in the box.

An enhancement factor $\beta_{a}$ for configuration $a$, may then be defined as follows:

$$
\beta_{a}=\frac{\left(n_{a}-\overline{n_{a}}\right)}{\sigma_{n_{a}}}
$$

where $n_{a}$ is the observed number of configurations $a$ existing in the refined box and $\overline{n_{a}}$ and $\sigma_{n_{a}}$ are defined by Eq. 18 and 19. The central limit theorem indicates that for a large number of iterations of a probability distribution, the distribution will tend to a normal distribution. The number of atoms in a large box model is sufficient to satisfy this condition. Consequently, the probability of a certain range of values of enhancement factors is that of a normal distribution. 
It follows that if, in a structural model, a configuration has an enhancement factor $\left|\beta_{a}\right| \geq 3$, this configuration is enhanced due to some ordering effect, and not merely the result of statistical noise in a random box. An enhancement in specific configurations can then indicate that the ordering is tending towards a certain long-range ordered structure. Reference tables of long-range ordered structures, and the Clapp configurations from which they are made, can be drawn up (see Supplementary Table S1).

The application of these enhancement factors to the analysis of order in a system can be demonstrated using the same simulated boxes as for the Warren-Cowley analysis (Section 3.3.1). The results are shown in Fig. 7. In the random case (a) the fluctuations are well within the bounds predicted probabilistically. For the statistically ordered case (b) a range of significant enhancements, both positive and negative, are observed. In contrast, with the micro-domain (c) and disperse models (d) there are massive enhancements in the configurations corresponding to the domains of the $\mathrm{Ll}_{2}(\mathrm{C}-1$ and $\mathrm{C} 16)$ and $\mathrm{L1}_{0}(\mathrm{C}-16)$ structures respectively. The Clapp configuration enhancement factors also identify enhancements even with the $5 \times 5 \times 5$ domain structures, making it an apparently more sensitive probe of the order present in the box than the Warren-Cowley parameter distributions.

If, as has been previously described, short-range order is considered to be an intermediate state between disorder and long-range order, there is likely to be much interest in the processes through which order develops. As the atoms diffuse through the solid forming the ordered structure it may be expected that not only the Clapp configurations present in the final structure will be enhanced, but also those that are structurally related. This may in turn highlight key structural motifs that are important to the process of formation of the final structure. 

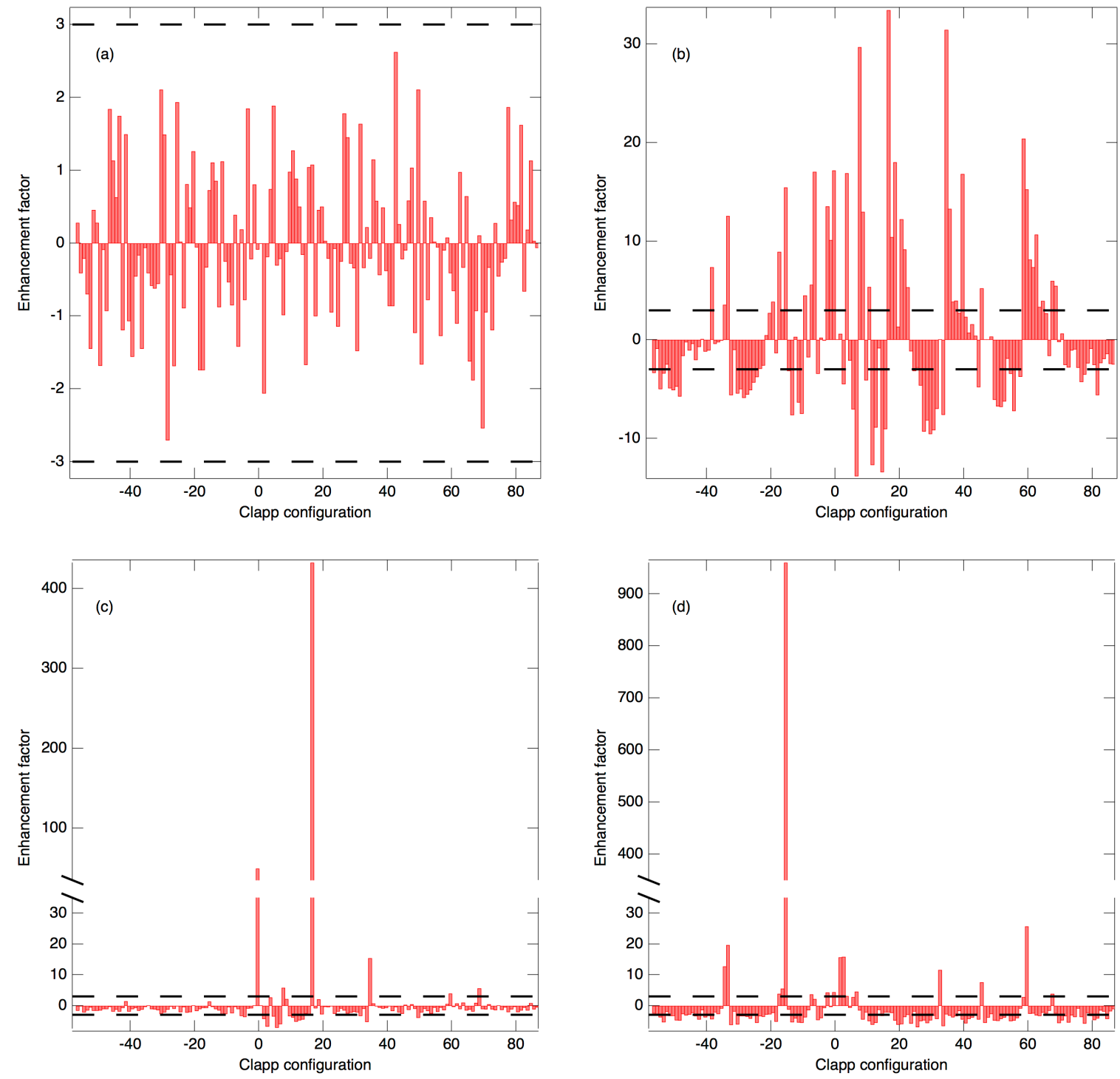

Fig. 7 - Enhancement factors for generated supercells of size 20x20x20 and stoichiometry $\mathrm{A}_{3} \mathrm{~B}$. The short range order present is as follows: (a) random, (b) statistical order created using a Monte Carlo simulation with a preference for dislike nearest-neighbour bonds (average $\alpha_{1}{ }^{\mathrm{AB}}=-0.0417$ and $\alpha_{2}{ }^{\mathrm{AB}}=0.1250$ ), (c) an $\mathrm{L} 1_{2}$ microdomain of size $10 \times 10 \times 10$ unit

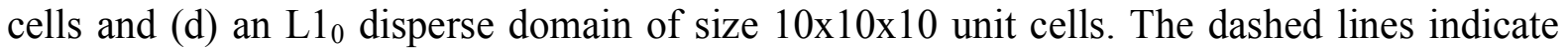
the bounds of the region $\left|\beta_{a}\right| \leq 3$ in which the enhancement may be due to random statistical fluctuations of the box rather than from ordering in the box.

An understanding of the relationship between the Clapp configurations is fairly easy to develop. For each configuration a consideration is made as to how the structure would be affected by either the addition, or removal of certain atomic types. This creates a degradation 
pathway that describes how the configurations would develop as the local composition of the box changes. In addition, many phase diagrams often identify a region containing a longrange ordered structure that extends beyond the ideal stoichiometry of the ordered structure. In these 'off-composition' alloys cases, the degradation pathways can also be used for identifying the type of ordering that might be present. Fig. 8 shows the degradation pathways related to the $\mathrm{C} 16$ configuration, an essential component of the $\mathrm{L} 1_{2}$ structure. Returning again to Fig. 7 in the microdomain case (c), the enhancement in the $\mathrm{C} 34$ configuration can now be understood due to it's degradation relationship to the C16 structure, as seen in Fig. 8. The full degradation pathway diagram for all the structures derived from a face centred cubic structure is too complex to reproduce here, but can be found in the supplementary information (Figure S4).

So far, only binary alloys have been considered in this analysis. Extensions of the Warren-Cowley parameters to multicomponent systems have already been discussed. The Clapp configurations, unfortunately, may not so easily be extended to higher dimensions. Whilst for a binary alloy the number of arrangements of two atom types in a face centred cubic lattice may be reduced by symmetry to the 144 already discussed, for a three-

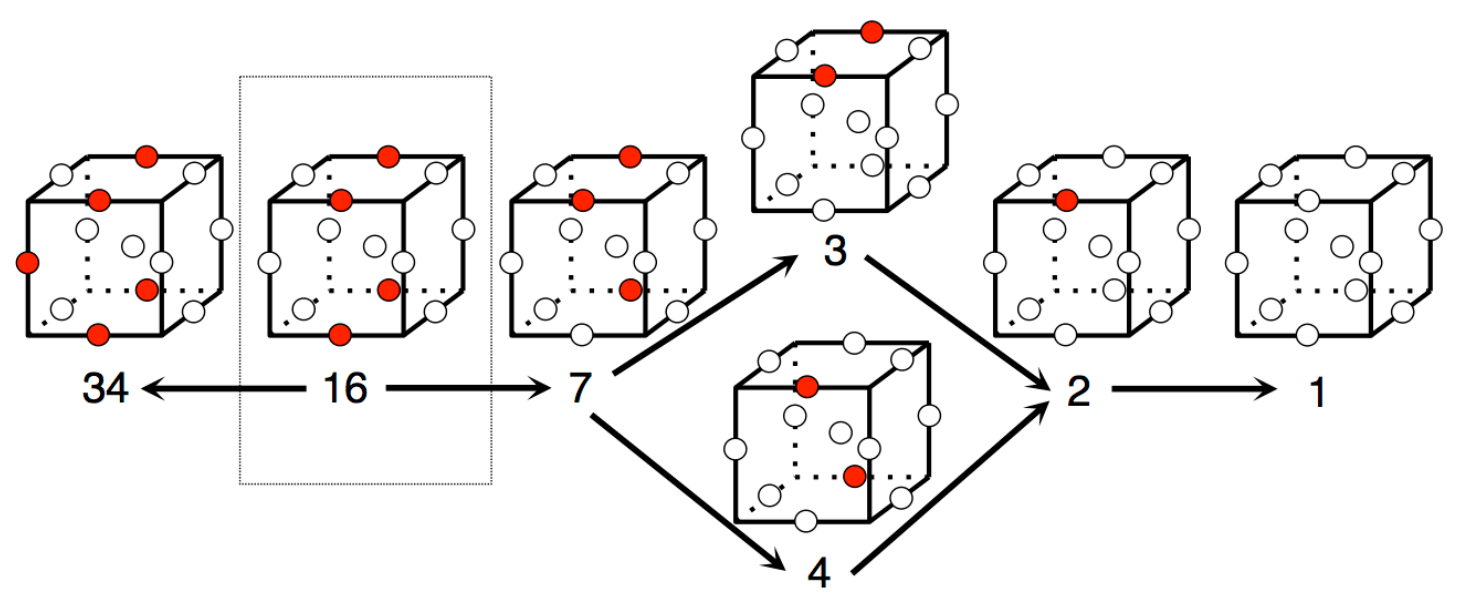

Fig. 8 - Degradation pathways for the C16 structure as concentration of B atoms (filled) in the structure is either increased or decreased. Numbers indicate the Clapp configuration. 
component system the same symmetry reduction yields 12,111 configurations. It is proposed, therefore, that Clapp configurations are only likely be of practical use if combinations of atoms are used to reduce the system to a pseudo-binary system. This will often make chemical sense due to the natural partitioning of elements onto specific sites during ordering transitions.

\section{Discussion and Conclusions}

It is clear from the preceding sections that the use of total scattering for the analysis of short-range order in crystalline alloys could provide valuable insight into the structure and properties of many complex alloy systems. To increase confidence in the results obtained in such a manner, it is essential to establish a systematic methodology for the collection and analysis of data.

\subsection{Proposed method for the analysis of short-range order in alloys}

The following method for the analysis of total scattering data from a alloy is proposed:

- Samples should be prepared in a manner which produces an adequate powder average.

- High quality total scattering data should be obtained using X-ray synchrotron and/or spallation neutron sources.

- Atomistic models representative of the long- and short-range order of the alloy, should be produced using appropriate simulation and/or refinement techniques, guided by the total scattering data.

- Short-range order in the system should then be determined, quantified and described by the methods outlined in this work:

i. Warren-Cowley distributions: to demonstrate the type and magnitude of order occurring in the system. 
ii. Clapp Configuration enhancement factors: to identify the local arrangement of atoms.

iii. Degradation pathways: to identify the key structural motifs and related LRO structures.

\subsection{Conclusions}

Theoretical work has suggested the use of the total scattering technique may provide new insight into ordering transitions and short-range ordering occurring in alloy systems. In the examples considered, the simulated boxes containing different types and amounts of shortrange order have been used to show that PDFs contain the necessary level of information to understand and differentiate between the different short-range order models. In addition, developments to the quantitative descriptions of short-range order used historically have extended their applicability to the analysis of order in large box models, such as those obtained from RMC refinements. Using a combination of Warren-Cowley parameter distributions, Clapp configuration enhancement factors and degradation pathways, a quantitative description of the ordering in the system can be provided. This level of quantitative information is required if models are to be developed to explain the link between the observed order and physical properties such as resistivity or strengthening.

It is anticipated that the proposed methodical approach to the measurement, fitting and analysis of short-range order using total scattering, will provide new insights into a host of alloy systems and ordering reactions.

\section{Acknowledgements}

This work was supported by the STFC ISIS Facility and the Rolls-Royce plc/EPSRC Strategic Partnership under EP/H022309/1 and EP/M005607/1. The authors gratefully acknowledge STFC for the provision of beamtime at Diamond Light Source Ltd. (EE10354, 
EE11665) and the ISIS Facility (RB1510579, RB1520332) and thank Dr. Stephen Hull for useful discussions.

Appendix A. Supplementary data

Supplementary data relating to this article can be found online.

$\underline{\text { References }}$

1 E.E. Stansbury, C.R. Brooks, T.L. Arledge. Specific-heat anomalies in solid solutions of chromium and molybednum in nickel - Evidence for short-range order. J. Inst. Met., 94, 4 (1966), 136.

2 Baer, G. Uberstruktur und K-Zustand im system nickel-chrom. Z. Metallkd., 49, 12 (1958), 614-622.

3 P.L. Rossiter, P. Wells. The dependence of the electrical resistivity on short-range order. J. Phys. C: Solid State Phys., 4 (1971), 354-363.

4 P.v.Blanckenhagen, B. Scheerer. Spin-wave dispersion relation in partially ordered $\mathrm{Ni}_{3}$ Mn. J. Magn. Magn. Mater., 13 (1979), 116-118.

5 Fisher, C.J. On the strength of solid solution alloys. Acta Metall., 2 (1954), 9-10.

6 Flinn, P.A. Solid Solution Strengthening. In Strengthening Mechanisms in Solids: Papers presented at a seminar of the American Society for Metals, October 13 and 14, 1960 (Metals Park, Ohio 1960), American Society for Metals, 17-50.

7 F. Pettinari-Sturmel, J. Douin, A. Coujou, N. Clement. Characterisation of short-range order using dislocations. Z. Metallkd., 97 (2006), 200-204.

8 Bragg, W.L. The diffraction of short electromagnetic waves by a crystal. Proc. Cambridge Philos. Soc., 17, 1 (1913), 43-57.

9 H.Y. Playford, L.R. Owen, I. Levin, M.G. Tucker. New insights into complex materials using Reverse Monte Carlo modeling. Annu. Rev. Mater. Res., 44 (2014), 429-449.

10 Debye, P. Dispersion of Rontegen rays. Ann. Phys., 46 (1915), 809-823.

11 Keen, D.A. A comparison of various commonly used correlation functions for describing total scattering. J. Appl. Crystallogr., 34 (2001), 172-177.

12 C.L. Farrow, P. Juhas, J.W. Lui, D. Bryndin, E.S. Bozin, J. Bloch, Th. Proffen, S.J.L. 
Billinge. PDFfit2 and PDFGui: computer programs for studying nanostructure in crystals. J. Phys.: Condens. Matter, 19, 33 (2007), 335219.

13 R.L. McGreevy, L. Pusztai. Reverse Monte Carlo simulation: A new technique for the determination of disordered structures. Mol. Simul., 1, 6 (1988), 359-367.

14 Soper, A.K. Tests of the empirical potential structure refinement method and a new method of application to neutron diffraction data on water. Mol. Phys., 99, 17 (2001), 1503-1516.

15 G.E.P. Box, M.E. Muller. A note on the generation of random normal deviates. Ann. Math. Stat., 29 (1958), 610-611.

16 M.G. Tucker, D.A. Keen, M.T. Dove, A.L Goodwin, Q.Hui. RMCProfile: reverse Monte Carlo for polycrystalline materials. J. Phys.: Condens. Matter, 19, 33 (2007).

17 O. Gereben, P. Jovari, L. Temleitner, L. Pusztai. A new version of the RMC++ Reverse Monte Carlo programme, aimed at investigating the stucture of covalent glasses. $J$. Optoelectron. Adv. Mater., 9, 10 (2007), 3021-3027.

18 O. Gereben, L. Pusztai. RMC_POT : A computer code for reverse Monte-Carlo modelling the structure of disordered systems containing molecules of arbitrary complexity. $J$. Comput. Chem., 33, 29 (2012), 2285-2291.

19 Klemm, W. Einge Probleme aus der Physik und der Chemie der Halbmetalle und der Merametalle. Angew. Chem., 62, 6 (1950).

20 Okamoto, H. Al-Ni (Aluminium-Nickel). J. Phase Equilib., 14, 2 (1993), 257-259.

21 L.Trieb, G.Veith. Kinetic Study of Short Range Order in alpha-CuAu alloys. Acta Metall., 26 (1978), 185-196.

22 B. Mozer, D.T. Keating, S.C. Moss. Neutron Measurement of Clustering in the Alloy CuNi. Phys. Rev. , 175, 3 (Nov 1968), 868-876.

23 B.D. Butler, J.B. Cohen. The structure of $\mathrm{Cu}_{3} \mathrm{Au}$ above the critical temperature. J. Appl. Phys., 65, 6 (1989), 2214.

24 Cowley, J.M. An approximate theory of order in alloys. Phys. Rev., 77, 5 (1950), 669-675.

25 Cowley, J.M. Short-Range Order and Long-Range Order Parameters. Phys. Rev., 138, 5A (1965), A1384-A1389.

26 B. Borie, C.J. Sparks. The interpretation of intensity distributions from disordered binary alloys. Acta Crystallogr., Sect. A: Found. Adv. , A27 (1971), 198. 
27 P. Georgopoulos, J. Cohen. The Determination of Short-Range Order and Local Atomic Displacements in Disordered Binary Solid Solutions. J. Phys., 12, 38 (1997), 191.

28 G.E. Ice, C.J. Sparks, A. Habenschuss, L.B. Shaffer. Anomalous X-ray scattering measurement of near-neighbour individual pair displacements and chemical order in $\mathrm{Fe}_{22.5} \mathrm{Ni}_{77.5}$. Phys. Rev. Lett., 68, 6 (1992), 863.

29 Fontaine, D. de. The number of independent pair-correlation functions in multicomponent systems. J. Appl. Crystallogr., 4, 15 (1971), 15-19.

30 A.V. Ceguerra, M.P. Moody, R.C. Powles, T.C. Peterson, R.K.W. Marceau, S.P. Ringer. Short-range order in multicomponent materials. Acta Crystallogr., Sect. A: Found. Crystallogr. , 68 (2012), 547-560.

31 Clapp, P.C. Atomic Configurations in Binary Alloys. Phys. Rev. B, 4, 2 (1971), 255-270.

32 Hart, G. Where are nature's missing structures? Nat. Mater., 6 (2007), 941-945.

33 B.E. Warren, H. Krutter, O. Morningstar. Fourier analysis of X-ray patterns of vitreous $\mathrm{SiO}_{2}$ and $\mathrm{B}_{2} \mathrm{O}_{3}$. J. Am. Ceram. Soc., 19 (1936), 202-206. 